\title{
To Restrict the Growth of Cancer and Convert it into a Cancer without Disease or Remission by Changing Dietary Patterns
}

Pandey $\mathrm{HM}^{1}$ and Nikhil Chaudhary ${ }^{2 *}$

${ }^{1}$ President, Madras Institute of Mind, Chennai, Tamil Nadu, India

${ }^{2}$ CEO, Madras Institute of Mind, Chennai, Tamil Nadu, India

\begin{abstract}
Abbreviations: AIF-1: Allograft Inflammatory Factor 1; ATP: Adenosine Tri Phosphate; BES: Base Excision Repair; COX-2: Cyclo Oxygenase 2; DHA: Docosahexaenoic Acid; DNA: Deoxy Ribonucleic Acid; DVT: Deep Vein Thrombosis; EPA: Eicosapentaenoic Acid; FAD: Flavin Adenine Dinucleotide; IGF1: Insulin Like Growth Factor 1;

KD: Ketogenic Diet; k-Ras: A gene that codes for Kirsten rat sarcoma viral oncogene homolog in Humans; MCHC: Mean Corpuscular Haemoglobin Concentration; $\mathrm{Mg}^{2+}$ : Magnesium; $\mathrm{MgSO}_{4}$ : Magnesium Sulphate/ Epsom Salt; MIP-2: Macrophage Inflammatory Protein 2; MMR: Mismatch Repair; MRI: Magnetic resonance imaging; NAD: Nicotinamide Adenine Dinucleotide; NER: Nucleotide Excision Repair; NF: b- Nuclear factor kB 22. NK Cells: Natural Killer Cells; OXPHOS: Oxidative Phosphorylation; PDH: Pyruvate Dehydrogenase Complex; PET CT: Positron emission tomography computed tomography; PTP: Permeability Transition Pore; PUJ: Pelvi-ureteric junction; RBC: Red Blood Cells; RNS: Reactive Nitrogen Species; ROS: Reactive Oxygen Species; TPP: Thiamine PyroPhosphate; UV DDBP- DNA-damage recognition protein; VEGF: Vascular Endothelial Growth Factor;

$\mathrm{v}$-Src: A gene found in Rous sarcoma virus that encodes a tyrosine kinase; VUJ: Vesico Ureteric Junction; WBC: White Blood Cells; XPDDNA-damage recognition protein; XPG- DNA-damage recognition protein
\end{abstract}

\section{Aim}

To restrict the growth of cancer and convert it into a cancer without disease or remission by changing dietary patterns in case of a single patient suffering from metastasized stage $4 \mathrm{~b}$ cervical carcinoma(recurrence or residual).

\section{Introduction}

No real progress has been made in the management of advanced or metastatic cancer for more than 40 years. Dr. William Li has achieved some success in prolonging the life of cancer patients by his antiangeogenic therapy (food) while Dr. Thomas N. seyfried has defeated cancer to a certain extent by his therapeutic fasting and ketogenic diet therapy. There are many more similar approaches used by experts throughout the world who have achieved some success against cancer.

In our case study we try to convert a case of recurrent metastasized hyper-metabolic cervical cancer into cancer without disease and ultimately to remission by using holistic nutritional therapy based on certain basic scientific principles and proven research. The patient who had little hopes to survive for more than a few days not only survived for 10 months but also showed remission clinically with improvement in overall quality of life.

Further research should be done by applying such therapies to find the underlying mechanism of cancer remission in general.

\section{Case Presentation}

We are not doctors to meet cancer patients every day. This lady of 42 years, $3000 \mathrm{kms}$ far, was introduced to us over the phone through an acquaintance when he had gone to meet her one last time because everyone expected her to die any moment. She had given up all medications and hopes of curing cancer. Since we are not medical professionals we worked within the framework of nutritional advice given to the patient by her doctors eat whatever you want to.

\section{Clinical history of the patient before the start of the therapy}

Cervical cancer was first identified in April 2013 with the mass extending into the left parametrium (not reaching the pelvic side walls) with involvement of upper 1/3rd of vagina; indenting left anterolateral wall of rectum without involving the rectum and indenting posterior wall of bladder without any obvious mucosal involvement [A1]. Patient was given concomitant Chemotherapy and Radiotherapy. By 22/7/2013, the patient was given chemotherapy + radiotherapy + Brachy Therapy along with one blood transfusion which was tolerated well and declared asymptomatic for cancer [A2].

On 11th September 2013, the patient was re admitted for complaint of difficulty in passing urine with lower abdomen pain and heaviness since 2 months. Diagnosis reveals recurrent/ residual cancer of cervix and lower uterine segment, predominantly involving anterior wall and protruding into endocervical canal and pericervical region. Also found to have hydronephrosis in left kidney and left ureteric opening could not be identified due to extensive tumour tissue and therefore stenting was not possible [A3]. Stent was installed in the right uretor for passage of urine and to save the kidney from collapsing. 16/9/2013, Oncologist mentions cancer to be stage III A and an inoperable case. During chemotherapy, patient suffered anaphylactic shock and was unable to tolerate certain drugs.

Whole Body PET CT Imaging report done on 4/10/2013 reveals Hypermetabolic cervix mass lesion is most likely mitotic and recurrence; hypermetabolic sternum and multiple rib lesions are likely metastatic; hypermetabolic right side oropharyngeal and lesion at the base of tongue. These were advised for further clinical correlation which were likely cancerous [A4].

The patient was given chemotherapy and discharged on 11/10/2013. After consulting various medical authorities personally the patient and

*Corresponding author: Nikhil Chaudhary, CEO, Madras Institute of Mind, New No. 5, Old No. 8, North Main Road, Anna Nagar West Extension, Chennai- 600101, Tamil Nadu, India, Tel: 9600099269; E-mail: nikhilchaudhary86@gmail.com

Received November 05, 2014; Accepted January 21, 2015; Published January 24, 2015

Citation: Pandey HM, Chaudhary N (2015) To Restrict the Growth of Cancer and Convert it into a Cancer without Disease or Remission by Changing Dietary Patterns. J Cancer Sci Ther 7: 019-030. doi:10.4172/1948-5956.1000320

Copyright: @ 2015 Pandey HM, et al. This is an open-access article distributed under the terms of the Creative Commons Attribution License, which permits unrestricted use, distribution, and reproduction in any medium, provided the original author and source are credited. 
her family decided to stop all treatments as they were advised that there was no treatment possible to save her life from the current condition of hypermetabolic metastasized cancer.

Shortly after the last treatment on $11 / 10 / 2013$, we got in touch with the patients family over the phone. We generally enquired about her condition and food habits. They had stopped all medication except a painkiller diclofenac (cataspa). We were also informed that she was almost bed ridden without any physical activity except going to the bathroom.

The foods regularly eaten by the patient were rice items, pulses, fruits, juices, tea, etc. The more focus was placed on giving her several glasses of fruit juices and easily digestible food like well cooked white rice, pulses, milk etc.

The patient admitted to have been drinking only approximately 1 glass of water every day.

We assured the family that we will try to help the patient in improving her health condition by way of changing her food habits and lifestyle which have proven basis for improving condition in cancer and overall health. Meanwhile we came to know from them that they were exploring the possibility of ayurvedic treatment from a local village vaidya.

The vaidya advised them certain homemade medicines to be taken with honey, sugar, ghee etc. He also advised not to consume citrus foods and curd. We tried to convince them that they should not go ahead with such advices as it will only deteriorate the condition of the patient and left the decision to them.

Initially we advised her in a mild way to stop eating refined and processed foods like juices, white rice etc and start with wholesome foods like vegetables, chapathi etc. These mild changes in the diet, made the patient feel better. This made them to take our advice more seriously. In the mean while we had also received her medical report and went through it.

After going through the medical report we prescribed the following diet chart and lifestyle changes which according to her, she started following fully since $26 / 12 / 2013$. On $26 / 12 / 2013$, her body weight was 62 kilograms. We advised her to start adding $\mathrm{MgSO}_{4}$ to her bathing water every day.

We advised her to fast on alternate days which she starts on $3 / 1 / 2014$. On the same day she started mild walking for the first time after many months.

On 5/1/2014 she travels $200 \mathrm{kms}$ by car with family and had a strainful journey. She got loose motions which got sorted out ultimately without any medication but later complained of increased pain in her abdomen and back.

$>$ On 8/1/2014 she walks for 20 minutes continuously which shows improvement in her physical condition as well as stamina.

$>$ On 15/1/2014 she informs about a white liquid discharge from her vagina which continued in the days to come but they didn $\mathrm{t}$ go for any medical check-up.

$>$ On 23/1/2014 she takes cannabis powder for the first time in replacement of her pain killer. She feels good and gets a sound and long sleep. But due to peer pressure and grown up children refuses to take it again.
$10 / 2 / 2014$ she travels again for $200 \mathrm{kms}$ by car to her native village to meet her family. Her brother informs us that her health looks much better and she feels confident and happy. They are all now hopeful of her survival. This definitely shows improvement in quality of her life. But again complains of abdominal pain.

$>13 / 2 / 2014$ she informs of feeling weak. But says she is mentally very strong and never deviated from her diet.

$>16 / 2 / 2014$ she informs that for the past about 15 days she has been feeling that the pain in her abdomen had subsided and also the pain was different. Earlier she felt something pressing and now it is a pricking pain with burning sensation like a fresh wound. She also reduced her dosage of painkiller to half.

$>17 / 2 / 2014$ for the first time after many months the patient says she feels very hungry and has temptation to eat a variety of food. She also informs us that she has started walking for 30 minutes and visits her neighbours in the evening while going for a walk. She also says she has now started taking curd.

$>18 / 2 / 2014$ she informs that the white fluid discharge is very high and she has to change her sanitary napkins regularly.

$>25 / 2 / 2014$ she says she has started mild form of exercise. By now her weight has reduced to $59 \mathrm{kgs}$.

$>11 / 3 / 2014$ she goes for a pilgrimage with a strainful travel of 60 $\mathrm{kms}$ and performs a ritual for 9 long hours. She complains of excessive pain in her abdominal area.

$>$ On 20/3/2014 she informs that her pain has increased and bleeding is present with redness in urine. Same night she informs that she is unable to pass urine.

$>21 / 3 / 2014$ she informs she passed urine in the morning and by the day no redness was present.

> 22/3/2014 MRI Scan [A5] of whole abdomen taken on our request-

I. The cancer has reduced to grade III B.

II. There is bilateral Hydrouretero Nephrosis.

III. Both Kidneys show grossly dilated pelvicalyceal system and both ureters.

There is a partial remission from $4 \mathrm{~B}$ to $3 \mathrm{~B}$. This partial remission mentioned in the report had instilled a greater confidence and satisfaction among the family members and the patient as well. The patient and family members developed a certain sense of trust in our approach of treating her.

$>26 / 3 / 2014$ she informs about swelling in the left leg with pain. This leads to complete bed rest for the coming 2 months. This was later diagnosed [A6] as DVT (Deep Vein Thrombosis) for which the doctor suggested treatments like surgery etc. which was not agreeable to the patients family.

> 12/4/2014 she informs of complete incontinence with continuous and excessive flow of urine all day. They consulted doctors but the incontinence could not be cured by any means.

$>16 / 4 / 2014$ swelling of the leg starts reducing.

> 16/5/2014 swelling has fully subsided and the patient starts walking mildly. 
4/06/2014 MRI scan taken at the same diagnostic centre [A7]

I. As compared with previous MRI dated 23-03-2014, the mass has mildly reduced in size.

I. Right hydroureteronephrosis has resolved. While the previous MRI had mentioned that there was bilateral Hydrouretero Nephrosis and Both Kidneys showed grossly dilated pelvicalyceal system and both ureters. Therefore we presume resolution of hydroureteronephrosis means no obstruction anywhere in the uretor and no undue dilation of either VUJ or PUJ.

II. Dilated left ureter till lower end, ballooned out renal pelvis and marked hydronephrosis due to infiltration of Vesico Ureteric Junction VUJ by mass (Associated PUJ obstruction cannot be ruled out). This shows reduction in mass as September 2013 findings indicated that left uretic opening could not be identified due to extensive tumour tissue mass.

III. Right ureter is not dilated. The previous MRI stated that there is involvement and encasement of bilateral vesicoureteric junction resulting in proximal dilation of both the ureters.

These results were achieved by putting the patient on a special diet and lifestyle which is described as follows:

The diet was designed to restrict the intake of refined carbohydrates, unwanted fats and oils, processed food, high calorie or low nutrient food to create energy stress in the body while providing all necessary nutrients possible. The patient was advised to fast alternate days but not to stay hungry. Therefore the diet planned is divided into two which includes a diet for the fasting day and a diet for the non-fasting day.

\section{- $\quad$ Diet for fasting day}

$>$ Start the day with a glass of freshly prepared wheat grass juice

$>2$ - 4 spoons of freshly ground flax seed totally per day

5-6 lemons in any form per day

> 2-3 cups of Green tea per day

$>$ Mix of powdered spices (Turmeric, Asafoetida, Ginger, Bishops weed, pepper, Sauf, Jeera, clove, cinnamon, cardamom) - 2 spoons per day

$>$ To take little Spirullina powder per day

$>1$ omega 3 capsule per day (EPA and DHA)

$>$ Lots of water.

- $\quad$ Non Dietary changes suggested (and followed)

$>20$ minutes of sunbath with maximum skin exposure during the morning hours

$>\mathrm{MgSO}_{4}$ to be added in bathing water

$>$ Mild walking couple of times every day with eventual increase in total time of walking

$>$ She was advised to meditate, keep calm, rest, sleep well, recite religious mantras and other activities to give mental peace.

\section{- Diet for Non fasting day}

$>$ Start the day with a glass of freshly prepared wheat grass juice

$>$ Breakfast consisted of only vegetable soup.- variety of green and coloured vegetables cut and boiled, eaten with the soup
$>$ Lunch consisted of Whole bran rice with a bowl of green chutney and vegetables

Dinner consisted of dry chapathi/ Whole bran rice with a bowl of chutney and vegetables

$>2$ - 4 spoons of freshly ground flax seed totally per day

5-6 lemons in any form like juice, with salad/ chutney etc per day

$>$ 2-3 cups of Green tea per day

$>$ Mix of powdered spices (Turmeric, Asafoetida, Ginger, Bishops weed, pepper, Sauf, Jeera, clove, cinnamon, cardamom) - 2 spoons per day

$>$ Raw salad with every meal

$>$ Little curd every day

$>$ To take little Spirullina powder per day

$>1$ Omega 3 Capsule per day (EPA and DHA)

$>$ Lots of water.

- Non Dietary changes suggested (and followed)

$>20$ minutes of sunbath with maximum skin exposure during the morning hours

> $\mathrm{MgSO}_{4}$ to be added in bathing water

$>$ Mild walking couple of times every day with eventual increase in total time of walking

$>$ She was advised to meditate, keep calm, rest, sleep well, recite religious mantras and other activities to give mental peace.

Strictly restricted food items included all types of refined carbohydrates (Sugar, maida, white rice), fruit juices, sweets, packaged food, fried food, re heated oil, over heated oil, processed food, over cooked food, milk and dairy except curd, starchy food etc.

The patient was advised to consume certain things like sprouts, almonds and walnuts whenever she felt like eating them but not to resort to the items which were advised to be strictly restricted. She was also advised not to eat when not hungry by following any time schedule. Also whenever hungry she should eat and not stay hungry. There was no restriction on the quantity of food and only on the type of food.

The patient followed the advice very strictly keeping aside all the hardships she faced. These advices were only suggestions made to her based on various scientific researches, books, etc and never forced on her.

\section{The problems we faced during the treatment}

The contact was only through phone, email and mail with the patient and her caregivers. Our physical absence made advising and monitoring difficult. We were in constant touch with the caregivers and patient over the phone at least 3-4 times a day. The patient was adamant on not eating certain things like garlic, Vitamin B Complex capsules etc. The patient initially refused to take lemon, gooseberries and citrus items and curd as she was advised by the herbal doctor (Vaidya). Only later on when she developed a lot of confidence in us, she discarded all the advices of the herbal doctor. The long distance travelling done by the patient which ultimately caused her injury. Replacing the pain 
killer with cannabis (it's available legally in the place of residence of the patient) which eventually she did not agree.

On 12-3-2014, the patient travels for a pilgrimage where she spends 9 long hours in a ceremony. We presume that the physical strain exerted by her caused her injury in her cervical region. After this she developed swelling in her left leg. Though we were told that this is normally what happens in cervical cancer patients we were confident that this was not related to cancer and was purely local effect due to injury. This was later diagnosed as Deep Vein Thrombosis. We presume this was due to injury caused to her veins because of physical overstraining during the travel and while performing ceremony there. Without any surgical procedure/ medication, DVT was resolved after 2 months. During this period the patient also developed incontinence and was rendered almost bed ridden. We presume that opening and clearing off of the left ureter from invasive tumor tissues and ballooning out of left renal pelvis resulted in incontinent behaviour of urine from the left kidney.

The patient still continued to take the painkiller diclofenac which almost led to her kidney failure and was admitted to the hospital for treatment of renal complications as urinary incontinence ultimately led to her inability to pass urine. There was also a point when the doctors were afraid that she might die due to kidney disease rather than cancer.

The painkiller being given to the patient was diclofenac. Diclofenac has been found to cause severe damage to kidneys in vivo in animals [1]. Moreover diclofenac is known to cause toxicity and also has antibacterial effects. Later when she was admitted to the hospital for urinary problems, the doctors immediately stopped the pain killer. We had also consulted a senior nephrologist in Chennai who reconfirmed that diclofenac cause kidney damage and suggested to stop it immediately and replace it with some other painkillers.

Cannabis has been a part of the human consumption from a very long time. Approximately 60 unique compounds known as cannabinoids have been identified. 9 tetrahydrocannabinol (THC) is the most studied and active compound identified. The biological effect of cannabis is due to similarity to endocannabinoids

Anandamide and 2-arachidonoylglycerol which bind and activate cannabinoid receptors [2].

Cannabis has been found to be anticarcinogenic, antiangiogenic, proapoptotic; antiemetic, apetite regulator, inducing anti-anxiety effects, pain reducing, has selective action towards tumor cells and is not harmful to normal cells [2-6]. The most important reason why cannabis was suggested to the patient in this case was because of the severe abdominal pain suffered by her.

The readiness in advising the patient to take cannabis came because there has not been any side effect found due to cannabis administration among patients at low doses. The high dose that causes toxicity is several grams per kilogram of body weight. Also there have been no lethal cases documented due to consumption of cannabis [2]. The family accepted to try cannabis because it was legally available at their place and also has been a known thing in their community. When the patient had taken cannabis for two days, she felt a lot of relief in pain and also had a long sound sleep. But unfortunately due to objection from her grown up children, she had to discontinue cannabis.

\section{Fasting}

Fasting was advised to the patient with an intention to lower circulating glucose and induces a stress condition on the tumor. It has been found that therapy which reduces the overall circulating glucose and energy rich substrate reduces growth and progression of numerous tumor types which include cancers of the mammary, brain, colon, pancreas, lung and prostate [7].

Blood glucose levels are affected more by the total amount of calories consumed. Blood glucose levels also are related to blood insulin levels. It has been found that higher insulin levels elevate glycolysis by enhancing glucose uptake in tumor cells. Fasting also helps in lowering blood insulin levels and also tumor promoting Insulin like Growth Factor (IGF-1) levels and Vascular Endothelial Growth Factor (VEGF) [7].

Experiments have shown that the prognosis of cancers with elevated blood glucose is very poor and there is a linear correlation of blood glucose and the growth of tumor in vivo [7].

Extensive studies have been done to show that reduced glucose availability reduces tumor growth, proliferation, angiogenesis and inflammation [7].

The above graph (Figure 1) shows experimentally that there is a linear correlation between glucose levels (Uptake) and size of tumor.

Tumor blood vessels express leakiness and immaturity while normal cells have strong and better blood vessels. It has been found that reduced caloric intake reduces the abnormal blood vessels and promotes the formation of necessary normal blood vessels [7].

Caloric restriction causes Tumor cell apoptosis and this phenomenon has been demonstrated experimentally. If energy from glycolysis is reduced, tumor cells will die [7].

Caloric restriction is anti-inflammatory as it reduces phosphorylation and degree of transcriptional activation of NF- B dependent genes COX-2 and AIF-1 in certain tumor and also reduced certain inflammatory markers like macrophage inflammatory protein2(MIP-2) [7]. It has also been found that the neutrophils show decrease in phagocytosis of pathogens with high sugar levels irrespective of the number of neutrophils themselves while the highest neutrophilic phagocytosis was seen after fasting [8].

Another interesting fact brought out by Dr. Thomas N Seyfried in his book Cancer as a metabolic disease is that under calorie restricted diet, glucose uptake is upregulated in normal cells while it is downregulated in cancer cells [7].

The glucose was monitored on a regular basis for the patient. The values are for fasting glucose with an exception that the patient drank wheat grass juice before taking the blood sample (Figure 2).

We were able to monitor the values only from 31st January 2014 by which time the patients glucose had already come to lower levels. The above graph clearly shows that the values were generally between 60 and 85 and fluctuated on a daily basis due to alternate day fasting and daily activities.

We presume the low values attained for blood glucose is one of the most important factors in achieving remission of cancer.

Glycolysis produces energy 100 times faster than Oxidative Phosphorylation (OXPHOS). Energy production during short energy intense workout is through glycolysis.

The enzyme required to convert pyruvate to Acetyl CoA is Pyruvate Dehydrogenase Complex (PDH). This enzyme is formed with 5 cofactors namely Thiamine PyroPhosphate (TPP), Lipoamide, Flavin Adenine Dinucleotide (FAD), Co enzyme A, Nicotinamide Adenine 


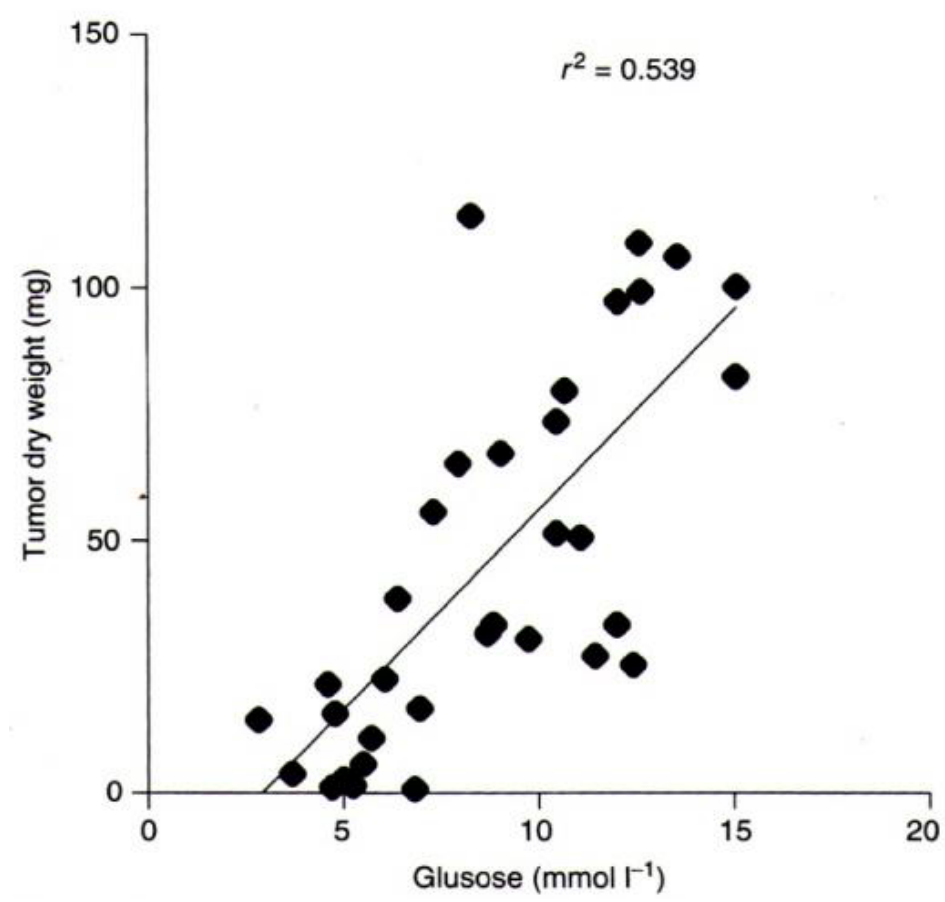

Figure 1: The above figure shows a linear relationship of sugar consumption and tumor growth.

Daily Fasting Blood Glucose $(\mathrm{mg} / \mathrm{dL})$

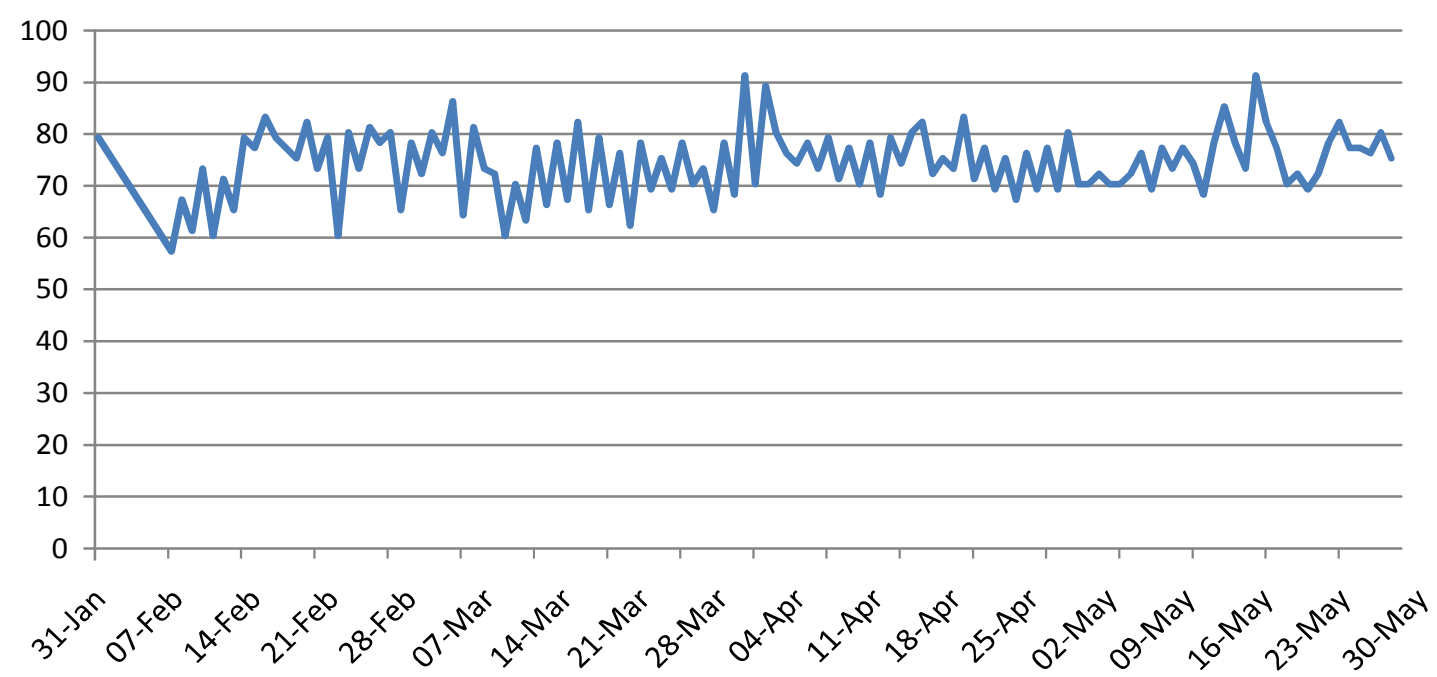

Figure 2: Fasting glucose levels from 31st January 2014 to 6 th June 2014 of the patient. The figure shows consistently the low levels of glucose during the treatment. The rise and fall in glucose levels are correlated to fasting and non-fasting days.

Dinucleotide (NAD+). These co factors in turn are formed with other biomolecules like Thiamine which is Vitamin B1, Sulpur, Flavin which comes from Riboflavin which is Vitamin B12, Pantothenate which is Vitamin B5, Cysteinie, Nicotinic acids which is Vitamin B3, purines etc. OxPhos can proceed only if all these necessary nutrients are present.

The diet designed by us had sources of abundant magnesium for the body

The incidence of hypomagnesemia in critically ill cancer patient is very high [9]. Magnesium repletion produced rapid disappearance of periosteal tumors [10,11]. Cancer cells and magnesium deficient cells show metabolic and physiological similarities in terms of cell membranes, Magnesium binding to membrane phospholipids, membrane permeability, membrane surface characteristics, membrane viscocity, Ionic flux changes etc. [12-18].

The ATP which is the Universal Energy Currency in biological forms is active only when it is coupled with $\mathrm{Mg}^{2+}$. $\mathrm{Mg}^{2+}$ is an important factor in almost every step of energy generation and other activities of 
cells. The genetic material and its stability are dependent on nutrients like $\mathrm{Mg}^{2+}$ Folic acid, etc.

Magnesium deficiency has been found in the cortex of the brain of patients suffering from Parkinson s disease. Cortex is the place where mitochondrial dysfunction has been observed in these patients. Similarly Magnesium deficiency has also been found in patients suffering from hypertension and diabetes mellitus [19].

The permeability transition pore (PTP) opening causes the depolarization of potential difference across the mitochondrial membrane. When PTP opens, cytochrome $\mathrm{C}$ is released which is a preprocess for apoptosis. It is also a known fact that depolarization causes increase in the Magnesium concentration of the cell. There may be a relationship between increase in Magnesium and apoptosis [19].

In nucleic acids magnesium acts as couterions to neutralize negatively charged phosphate groups. Magnesium is involved in the activation of the enzymes necessary for the DNA repair, Replication (topoisomerase II, polymerase I) and Transcription (rybonuclease $\mathrm{H}$ ). Magnesium also acts as a co factor for ribozymes. It is also necessary for the protein synthesis and formation of polysomes [20].

Damage caused to the DNA by both exogenous factors (Ultraviolet or electromagnetic radiation, high temperatures, viruses, polycyclic aromatic hydrocarbons, radiotherapy, chemotherapy etc) and endogenous factors (ROS and RNS) are repaired by the different DNA repair systems developed within the nucleus [20]. Nucleotide excision repair (NER), Base excision repair (BER), Mismatch Repair (MMR) are these systems. The damage caused by these endogenous and exogenous factors lead to mutations and process of carcinogenesis [20]. It has been found that magnesium acts as a cofactor at every step of NER and is impaired in the absence as well as high concentration of magnesium. The various DNA damage recognition proteins like UVDDBP, XPD and XPG are all magnesium dependent [20]. Magnesium is also important for the production of glutathione which quenches the ROS and RNS [20].

Magnesium is known to activate over 300 different enzymes in the body which include processes of transformation of proteins, lipids, carbohydrates, nucleic acids, electrolyte transportation etc. Magnesium also plays a vital role in the process of glycolysis, Krebs cycle, oxidation, electro coupling reactions etc. Alterations in the cellular magnesium concentration are reflected in the mitochondrial respiration rate as well [20].

Similarly one other vital nutrient of the body is Iron. Iron is a co factor for several enzymes which take part in cellular respiration and various metabolic processes like regulation of enzymes of citric acid cycle like aconitase, citrate synthase, isocitrate dehydrogenase, DNA synthesis, normal cell growth and proliferation, carries oxygen in the body, is crucial for normal mitochondrial electron transport, etc.

Since iron plays such an important and wide role in functioning and maintenance of cells that the requirement of iron in cancerous cells is high due to their rapid growth and proliferation.

Many scientists believe that since iron plays such a major role in the growth and proliferation of cancer cells, there is a significant possibility that reduction of iron may show anti proliferative effect on tumor cells. Several studies have been conducted using different iron chelators to reduce the total iron content of the cells and it has been demonstrated that iron depletion induces apoptosis in these cells [21].

Certain reports also mention that iron reduction by phlebotomy decreased cancer risk in a supposedly normal population [22].
Let us consider that iron reduction can have anti proliferative properties and also be a therapeutic way to treat cancer. Still the big question remains that how will we achieve reduction of iron in cancer cells while keeping the supply of this vital nutrient intact for normal cells?

We never aimed at reducing iron from the body of our patient. Neither we are concluding anything from the limited data available to us. But, we would like to share some reports we gathered during the treatment which gave us amazing insights about the healing power of the human body.

This data shows that RBC and Haemoglobin concentration reduced with time while the Haemoglobin concentration per cell, MCHC, was always maintained within the normal range. This implies that the overall iron levels went down while the required iron levels were available (Figures 3-5).

The most interesting fact about the iron homeostasis here is that the food consumed by the patient all along consisted of rich sources of iron.

Therefore we can hypothesize that if a person, normal or cancer patient eats natural vegetarian food, iron will never create any imbalance or associated problems.

This also calls on the scientific and medical community to establish separate ranges and guidelines for normal people and cancer patients keeping in mind the individuality.

\section{Vitamin C}

Vitamin $\mathrm{C}$ has a wide therapeutic dosage range and an even wider safety margin (up to 100-200 grams daily safely). Many studies have established that vitamin $\mathrm{C}$ intake is inversely related to cancer, with protective effects shown for cancers of the pancreas, oral cavity, stomach, cervix, rectum, breast and lung etc. immune-stimulating action of the vitamin $\mathrm{c}$ is evident as Splenic and thymic weights of the vitamin-treated animals were higher than those receiving cytotoxic treatment alone.

In some cases, 'rebound effect' i.e. high dosage of intravenous vitamin $\mathrm{C}$ can cause symptoms of ascorbate deficiency is seen and can be avoided by concurrently supplementing with oral doses of vitamin $\mathrm{C}$ (and possibly bioflavonoids and other antioxidants). In our case the patient was only given natural sources of Vitamin C.

Vitamin C is toxic to several cancer lines at doses that are non-toxic to normal cells. It was found that at a dose of $7.04 \mathrm{mg} / \mathrm{dl}$, vitamin $\mathrm{C}$ is completely toxic to cancer cells while being completely non-toxic to normal cells [23]

Linus Pauling and Ewan Cameron reported that patients treated with high doses of vitamin $\mathrm{C}$ had survived three to four times longer than similar patients who did not receive vitamin C supplements. Dr Cameron treated 100 advanced cancer patients with 10,000 milligrams of vitamin $C$ per day and compared with that of 1,000 patients who had received no vitamin C. Moreover, the vitamin C patients were said to have shown an improvement in their quality of life. Pauling later contributed further evidence of vitamin C's efficacy in mammary tumours in mice. Furthermore, Riordan, from the Bio-Communications Research Institute, and Jackson have reported on cases of cancer successfully treated with intravenous vitamin C therapy. The Journal of Oncology reported two cases of complete cancer regression in response to high-dose ascorbic acid therapy. 


\section{Red Blood Cells Total}

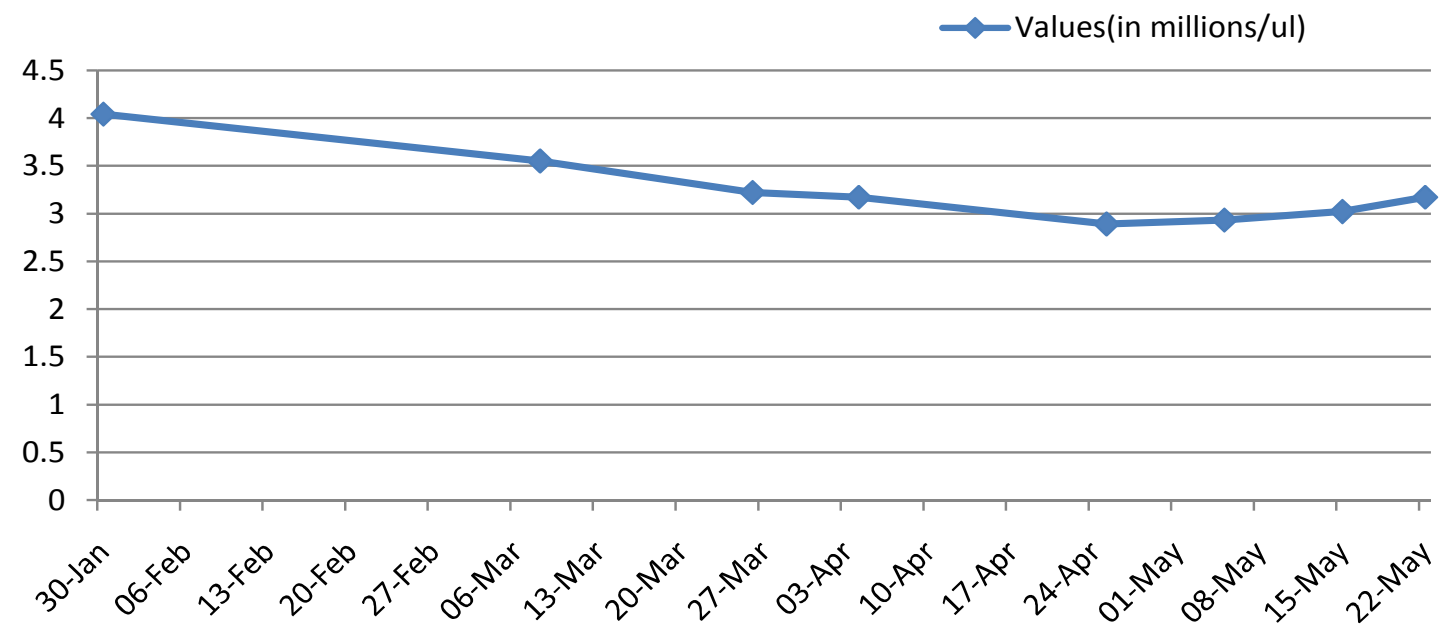

Figure 3: Red Blood Cells count taken during the treatment period of the patient.

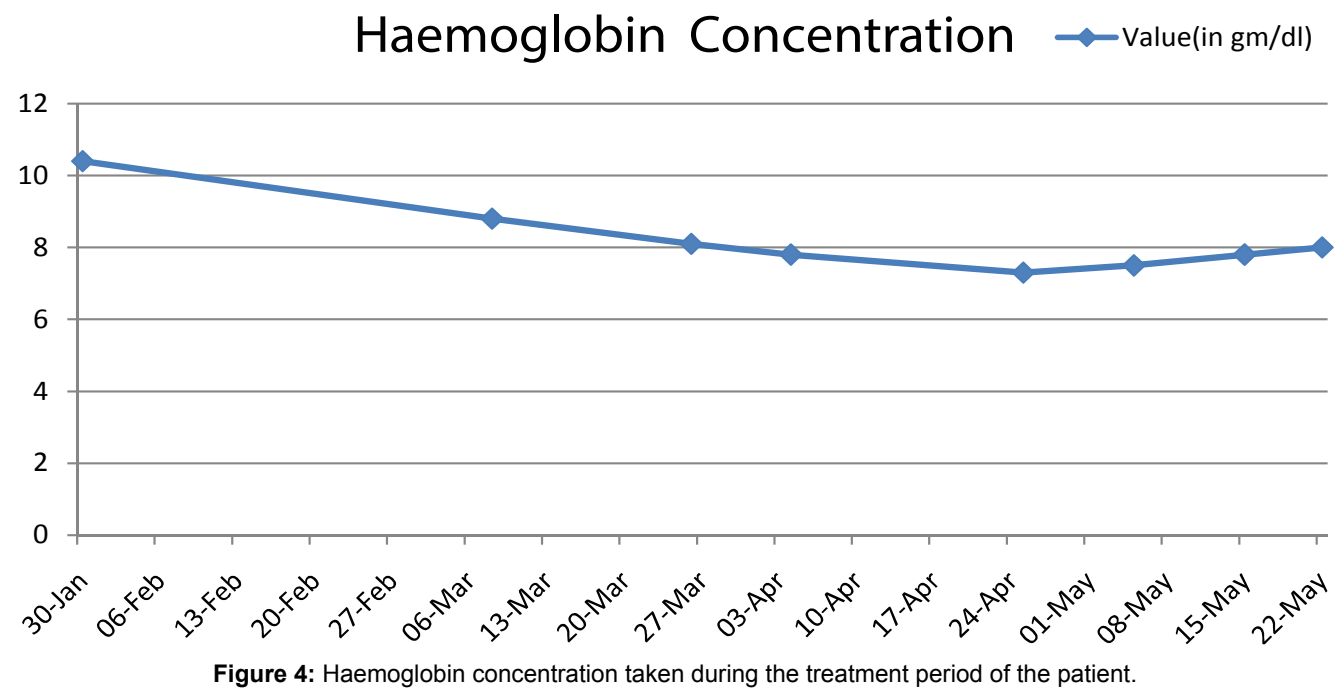

Mean Corpuscular Haemoglobin Concentration

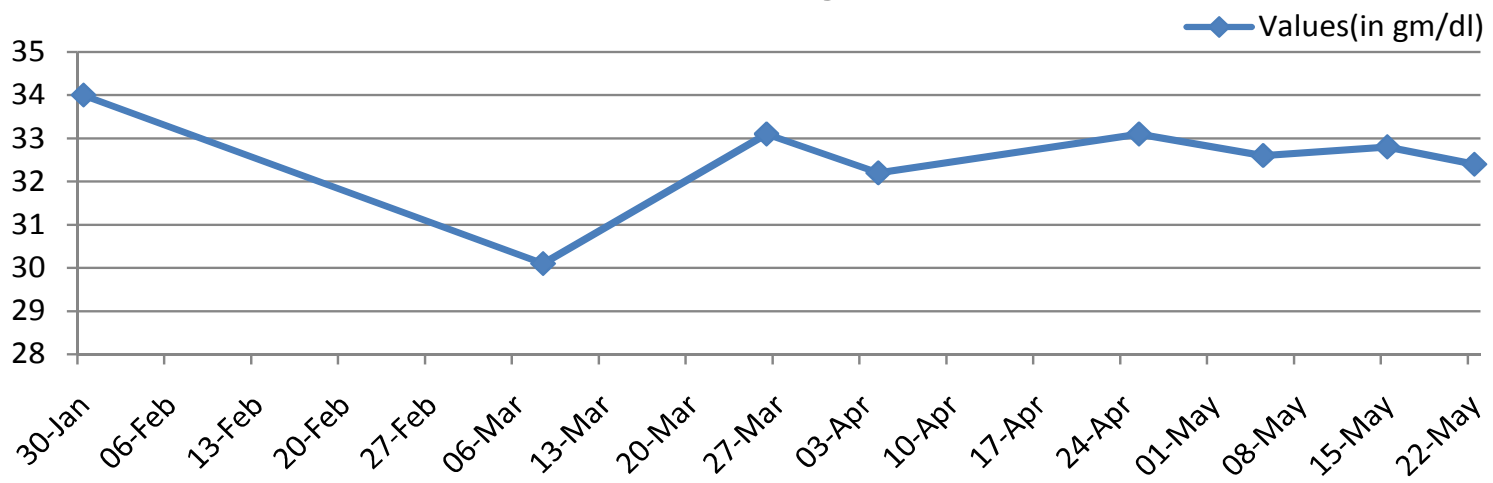

Figure 5: Mean Corpuscular Haemogobin Concentration values taken during the treatment period of the patient. 
Vitamin C is so important because it is considered the main redox stabilizer, stabilizing oxidation reactions in the body. The antioxidant capacity is directly linked to the effectiveness of the immune system and inflammatory resolution [23].

Therefore we introduced lemon and other vitamin $\mathrm{C}$ rich foods in her diet.

\section{Inflammation, immunity and redox state}

RudolfVirchow was the first to establish a linkbetween inflammation and cancer when he found that leukocytes were present within tumors and certain patients had developed cancer at the exact same spot where they received a blow or repeatedly got rubbed by something like a shoe or a tool. Dvorak referred to tumors as Wounds: that do not heal because he found that the causes of various cancers are also the cause of inflammation [24]. Skin Inflammation caused by ultra violet light associates to Melanoma, Inflammation due to hepatitis associates to hepatocellular carcinoma, Lung inflammation during bronchitis due to silica/ asbestos/ smoking associates to lung cancer, inflammation caused by reflux oesophagitis is associated with oesophageal carcinoma etc [23]. Role of inflammation in carcinogenesis is widely accepted and a lot of research has been done in the past decade in this area. Even dominant oncogenes such as $\mathrm{v}$-Src and K-Ras have been found ineffective in inducing cancer without injury and subsequent tissue regeneration [25].

Chronic inflammation plays an active role in all the stages of carcinogenesis namely initiation, promotion and progression.

Anigiogenesis is the formation of blood vessels for tumor growth. Degree of vascularity determines the invasiveness and malignancy of tumors and also prognosis is better for tumors that are less vascular [19]. The inflammatory response promotes neoangeogenesis to provide the fast growing cancer cells with nutrients for growth and proliferation [25]. Angiogenesis is the event that separates the two forms of cancer namely the preinvasive and dormant form from the invasive and metastatic phases where the cancer is hyper metabolic [26].

This implies that the immune cells, inflammation, angiogenesis are all an integral part of cancer initiation, promotion and progression.

The role of inflammation in cancer is dual and it acts as both pro-tumorogenic as well as anti tumorogenic which is decided only by the environmental and microenvironmental conditions where the tumor is present. $T$ cells as determined by their effector functions and neutrophils depending on their differentiation status can exert both tumoricidal and tumor promoting functions. Therefore even the cancer therapy like chemo therapy and radiation can induce either tumor suppressing or promoting responses [25].

Cancer chemo prevention is defined as the use of pharmacological, natural, or dietary agents to inhibit the development of invasive cancer by blocking DNA damage caused by carcinogens or by arresting the progression of pre malignant cells after damage has already occurred [27].

William Li with his group has worked intensively on anti angiogenic properties of various foods. He has shown both in vitro and in vivo the anti angiogenic effect of certain compounds present in food items. These include Epigallocatechin-3-gallate (EGCG) present in green tea, Genistein present in Soybeans, Resveratrol present in grapes, wines, peanuts, berries, etc, Lycopene present in tomatoes and coloured fruits, Omega -3 Polyunsaturated fatty acids which also includes docosahexaenoic acid (DHA) and eicosapentaenoic acid (EPA) found in cold water fishes, fish oil, walnuts, flax seeds etc, Glucosinolates, Isothiocynates and Indole-3-carbinol which are found in Cruciferous vegetables, Flavonoids which are a family of polyphenols and are plant pigments found in almost all vegetables like spinach, onions, parsley, beets, thyme, lettuce, chicory, argula, red lettuce etc, other flavonoids like quercetin, anthocyanidins, proanthocyanidins, ellagitannins etc are found in herbs, spices, fruits and vegetables like berries, grapes, cinnamon, cacao, etc. One important flavonoid is curcumin found in turmeric. Other novel dietary inhibitors showing anti angiogenic acitivities identified are phytoalexin called brassinin found in Chinese cabbage, citrus derived bioflavonoids hesperidin and narigenin, ellagic acid from berries, pomegranate and other fruits, silymarin from milk thistle and artichoke and organosulfur allyl disulfide from garlic. $\mathrm{He}$ also mentions in his paper about curcumin showing induction of apoptosis and other antiangiogenic properties [26].

Even the great neuro scientist Dr. David Servan Schrieber in his celebrated book Anti-Cancer-a new way of life has mentioned a lot about foods which have anti-cancer properties. Here he talks about green tea, turmeric, ginger, cruciferous vegetables, garlic, onions, leeks, shallots, chives, carotenoid rich fruits and vegetables, tomatoes, soy, mushrooms, herbs and spices, seaweeds, omega $3 \mathrm{~s}$, selenium rich foods, Vitamin D, probiotics, berries, citrus fruits, pomegranate, red wine, dark chocolate, mushrooms, etc. He tells us about the various effects of these foods on our body, immune system and healing [24].

There are many epidemiological studies conducted by scientists throughout the world to establish a relationship between diet and occurrence of cancer. One of the greatest and largest study ever conducted is the well known The China Study by Dr. T. Colin Campbell and his team [28].

What is more intriguing is the fact that a substance like curcumin is eaten in India in every household and almost by every individual on a daily basis. Most of these foods are a daily part of our life. But if they have preventive and curative properties then the obvious doubt arising in our mind will be why people in India and Asian countries getting cancer are?

We believe that the answer is processing and balance. It is not one single ingredient or factor but the whole system which is at work either for remission or genesis of cancer.

Though $\mathrm{T}$ cells are known to target and kill cancer cells, Fais and collegues showed that malignant melanoma cells eat $\mathrm{T}$ cells and certain tumor cells eat even NK cells [7]. In such situations therapies induced to just increase the immune cells may not help. Here the therapy required will be to change the characteristics of tumor cells and immune cells rather than just cloning immune cells and injecting them.

It has also been found that certain aggressive cancer cells after ingesting yeast cells commit apoptosis. This characteristic of cancer cells of committing apoptosis after phagocytosis can be a very effective treatment if the substrate for ingestion is identified [7].

In this case we found that the natural diet increased the immune cells by approximately $50 \%$. As we have already mentioned earlier the phagocytic activity of the neutrophils goes down by consumption of sugar and increases with fasting. We believe similar affect would be taking place in boosting the immune response of immune cells by consuming natural whole foods (Figures 6 and 7).

We also monitored Total Cholesterol levels and found that there was a general reduction in total Cholesterol levels. We assume that the 
reduction of total cholesterol is associated with better production of cellular energy.

\section{Normal Cells vs. Cancer Cells: The Genetic Makeup}

There has been a lot of controversy on the genetic theory of cancer. Plethora of research has evolved to hypothesize the genetic evolution of cancer and similarly huge amount of research data points to the fact that cancer does not have genetic evolution.

But there has been consensus among most scientists that cancer cells show genetic variations, they are not well differentiated, have varying number of mutations etc. Scientists also suggest that cells differ genetically in different tumors. What is interesting to know is that any two cells of the same tumor also show wild variations among themselves. As Dr. Seyfried says Genetic heterogeneity and randomness is the norm rather the exception for mutations found in most sporadic cancers [7].

It has also been shown that when the nucleus of a cancer cell is transplanted in a normal cell, they beget normal cells while the nucleus of a normal cell in the cytoplasm of a cancer cell begets cancer cells [7].

Dr. Colin Campbell research, The China Study which is the biggest epidemiological study ever conducted has shown that the genetic disposition to cancer in any person accounts for only $2-3 \%$ of the total cases [28].

\section{Results and Summary}

Our main attempt was to reduce the tumor mass. Therefore as and when possible we have taken MRI to monitor the state of growth/ remission of the tumor mass. We were inclined to take only MRI scan and not PET CT scan because of glucose complication. In the discharge summary of $22 / 7 / 2013$, the doctor mentions the patient is asymptomatic of cancer [A2]. As on 4-10-2013 [A4] the PET scan reveals that the mass had metastasized upto

1) oropharyngeal and base of tongue

2) Sternum

3) Ribs

The patient confirmed the presence of lesions below the tongue. As mentioned earlier we started advising her on healthy diet and lifestyle and slowly gained her confidence. She started feeling better and in the meanwhile we collected all the necessary information from her. Thereafter as mentioned above we gave a comprehensive in detail diet

White Blood Cells Concentration

$\sim$ Values(in ths/ul)

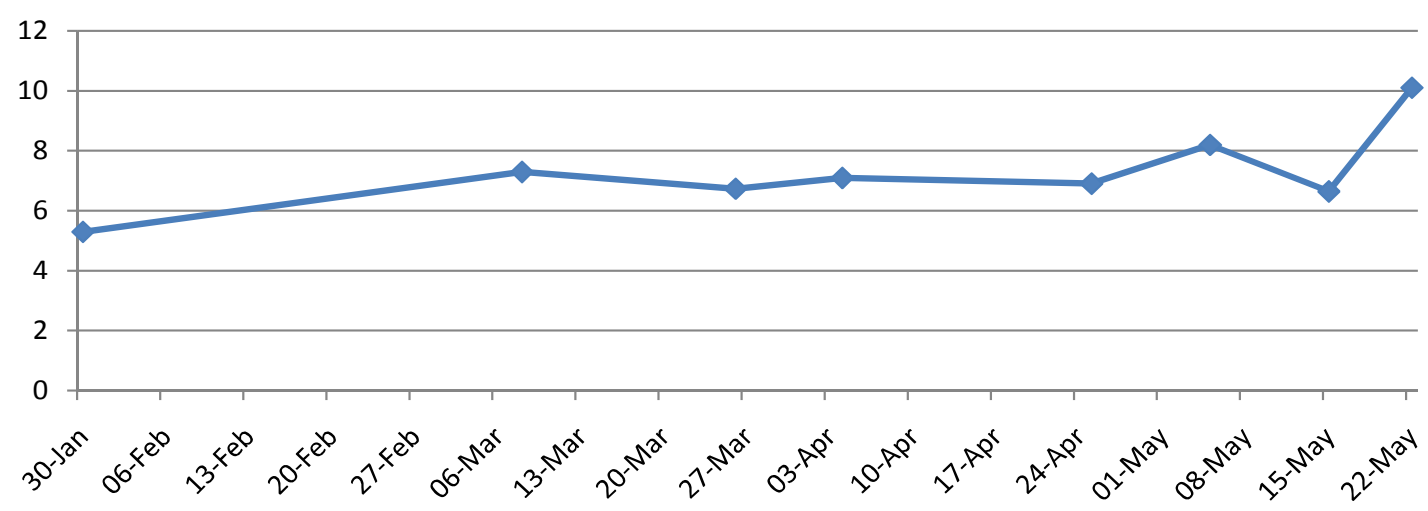

Figure 6: The White Blood Cells count observed in the patient during the course of treatment.

Total Cholestrol
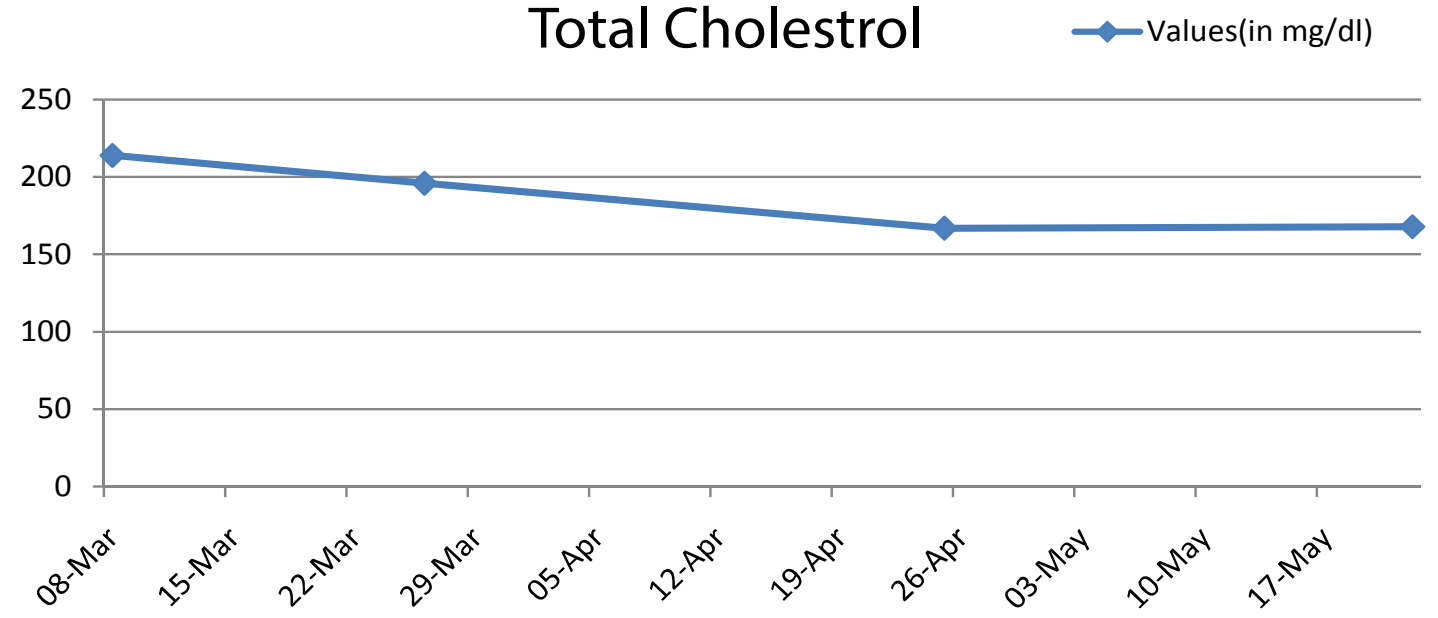

Figure 7: The total Cholesterol values taken during the period of treatment shows reduction. 
chart to be followed strictly. The patient adhered to the diet chart and lifestyle changes.

On 22/3/2014 another MRI was taken:

I. The cancer has reduced to grade III B.

II. There is bilateral Hydrouretero Nephrosis.

III. Both Kidneys show grossly dilated pelvicalyceal system and both ureters.

IV. Both VUJ (Vesico Ureteric Junctions) were surrounded by tumor mass and at both the junction points ureters were dilated.

There is a partial remission from $4 \mathrm{~B}$ to $3 \mathrm{~B}$. This MRI reveals that the left ureter was dilated whereas the findings as per the earlier scans taken in the middle of September 2013 was that the left ureteric opening could not be identified due to extensive tumour tissue. This suggests that there has been considerable reduction in tumor mass during this interval.

On 4th of June 2014, another MRI was taken in the same diagnostic centre. The findings revealed the following parameters:

II. As Compared With Previous MRI Dated 23-03-2014, The Mass Has Mildly Reduced In Size.

III. Right Hydroureteronephrosis has resolved and there is No Dilation of Right Uretor

While the previous MRI had mentioned that there was bilateral Hydrouretero Nephrosis and Both Kidneys showed grossly dilated pelvicalyceal system and both ureters. Therefore we presume resolution of right hydroureteronephrosis means no obstruction anywhere in the right uretor and no undue dilation of either right VUJ or right PUJ.

IV. Dilated left ureter till lower end, ballooned out renal pelvis and marked hydronephrosis due to infiltration of Vesico Ureteric Junction VUJ by mass (Associated PUJ obstruction cannot be ruled out).

This shows reduction in mass as September 2013 findings indicated that left ureteric opening could not be identified due to extensive tumour tissue mass.

V. Right ureter is not dilated. The previous MRI stated that there is involvement and encasement of bilateral vesicoureteric junction resulting in proximal dilation of both the ureters.

Thus there has been a tremendous improvement in the remission of tumor mass between 4-10-2013 [A4] and 4-6-2014 [A7].

We have also followed her blood parameters like Blood Glucose, Differential Blood Count, Liver function test, Kidney function test, Lipid Profile and also urine parameters regularly. The results obtained were fascinating and correlations with remission of cancer should be established by the scientific community for better prognosis and treatment of cancer in future.

During this treatment her quality of life also improved. Initially she was immobile, uncomfortable and suffered great pain. During the treatment she informed us about feeling light, walking without any trouble, reduced abdominal pain and also visiting neighbours and parents staying $200 \mathrm{kms}$ away. People around her also felt the improvement in her health and lifestyle as given above. She was also able to reduce the dosage of her painkiller by half.

Her quality of life was affected because of her strainful journeys and ceremony in the temple which eventually resulted in injury to her left leg. Though her problem was sorted out within 2 months but she had to suffer considerably during her recovery from the injury.

As revealed in the last MRI taken in June 2014, the kidneys especially the left kidney was badly affected. We presumed that the painkiller diclofenac was responsible for kidney damage which was later confirmed by nephrologists in Chennai.

Due to considerable reduction of tumor mass, the left side kidney and ureter got cleared and the passage of urine from left kidney became unhindered. This we presume was the main reason for urinary incontinence which affected her quality of life.

We also presume that due to certain blockages somewhere in the urinary system incontinence got converted into total stoppage of urine. This was repeated few times during which she had to get medical assistance.

She was admitted for urinary incontinence and complete stoppage of urine. She was treated by medical experts for a couple of days. During her treatment she was given blood transfusion and IV fluids. Her painkiller was also discontinued. During the stay in the hospital her diet also could not be followed.

\section{Discussion}

The case study presented here not only shows that the patient was able to achieve remission in cancer but also points out to the facts that cancer is a disease of altered metabolism like diabetes, hypertension, etc. It also proves that cancer can be treated and quality of life improved even in extreme cases. This treatment can be used to treat all types of cancers and not just cervical cancer with minor variations depending on individual cases and their body responses.

\section{Underlying mechanism of carcinogenesis}

We hypothesize that the cause for cancer is combination of the following aspects:

\section{Nutritional imbalance}

a. Refined carbohydrate and energy dense food: As explained above the abundance of glucose in cells hampers energy production in mitochondria. In fact abundance of glucose leads to glycolysis outside mitochondria and heat generation within mitochondria. According to our hypothesis this glycolysis and heat generation is most important contributory factor to the development of cancer. Without abundant supply of glucose the cancer cells are not able to command unlimited supply of energy and their faster and faster proliferation.

Refined carbohydrate and energy dense food lead to gut dysbiosis. It has been found that gut dysbiosis leads to enormous toxins within the circulatory system which in turn poisons each and every cell. In such a scenario the detoxification of cells becomes an impossibility resulting in inflexible functioning at cellular and mitochondrial levels.

b. Deficiency of vital nutrients, minerals, vitamins, phytonutrients etc: The burning takes place in mitochondria if pyruvate gets converted into acetyl CoA which intiates the krebs cycle. For this conversion to take place certain vital nutrients like Vitamin B1, B3, B5, Magnesium etc are absolutely necessary. In the absence of these vital nutrients the initiation of krebs cycle in the mitochondria simply does not arise. Recent researches show that mitochondria act as a store house of magnesium in the cell and are absolutely necessary for the functioning of mitochondria. It is necessary that toxin generated during various cellular processes are excreted from the cell then and 
there. If these toxins are not excreted then and there, they may hamper the entire energy production process in the cell. This excretion needs adequate cellular hydration. Even the deficiency of any one of the vital nutrients is enough to sub optimise energy production in the mitochondria and thus be a cause for glycolysis and therefore cancer.

c. Deficiency of adequate quantity of anti-oxidants to quench free radicals generated during energy production: The deficiency of antioxidants leads to accumulation of free radicals in the mitochondria. This reduces the production of ATP inside the mitochondria automatically shifting the burden of energy production to glycolysis in the cell which does not produce any free radical normally.

\section{Mismatch between energy produced and energy needed on a continuous basis}

If on a continuous basis there is a deficit of energy, it will prompt the system to switch to glycolytic process on a permanent basis. This becomes the main reason for promotion and progression of cancer. This deficit sometimes could be more acute locally and therefore the initiation and promotion will take place in those organs. Increased energy is necessary to maintain a certain potential difference across cellular membrane, efficiency of cellular reactions, mental stress, anxiety leading to cortisol production, wound healing etc.

We know from our experience that metabolic diseases arise due to reasons enumerated above under aspect 1. If aspect No. 2 is also satisfied then the promotion and progression of cancer takes place. Whether the cancer cells will be benign or aggressive and metastatic is also decided by the above mentioned aspects No. 1 and 2 .

\section{Et $=$ Total Energy Production}

Emit $=$ Mitochondrial energy production (ATP)

Efer $=$ Energy production by fermentation (Glycolysis)

$\mathrm{X}=$ Lower threshold of Efer

$\mathrm{Et}=\mathrm{Emit}+\mathrm{E}$ fer

Efer $=0$

Under normal circumstances,

Efer $=0$ (generally)

Cancer takes place when,

Efer $>$ X (Always)

Hypothesis for Cancer Treatment:

If, $X=0$

Cancer can be cured.

To achieve this

Et $=$ Emit

This can be achieved by two ways

1) Reduce Et

2) Increase Emit.

In other words, cancer can be cured by combining the following aspects

a) Restriction on consumption of energy dense food: Restricted energy supply to the system will create uneven level playing field to cancerous and normal cells. When there is a restricted energy supply, the glucose transporters in the cancer cells are down regulated while those in normal cells are unregulated. Therefore in case of energy scarcity, cancer cells starve and normal cells thrive.

This type of food has also got implications in setting right gut dysbiosis and in turn resulting in better detoxification of cells and better immunity.

b) Replenishing vital nutrients, minerals, vitamins, antioxidants, phytonutrients etc: By providing vital nutrients, minerals, vitamins, antioxidants phytonutrients etc. through natural means as far as possible, energy production in the mitochondria can be enhanced. This will naturally lead to reduction in energy production by glycolysis to its minimum generally. This will also make the immune system stronger.

c) Reduction of total energy requirement: The total energy requirement can be reduced if physical and mental energy is deployed more efficiently.

We have followed the above mentioned principles and were able to achieve partial remission in stage $4 \mathrm{~B}$ highly aggressive hyper-metabolic metastasized cervical cancers. We are also confident that these results can be replicated in any number of cancer patients.

\section{References}

1. Seyfried TN (2012) Cancer as a metabolic disease: On the origin, Management and Prevention of Cancer. A John Wiley 8: Sons, Inc.

2. Dr. Colin Campbell; The China Study.

3. Kubota T, Shindo Y, Tokuno K, Komatsu H, Ogawa H, et al. (2005) Mitochondria are intracellular magnesium stores: investigation by simultaneous fluorescent imagings in PC12 cells. Biochim Biophys Acta 1744: 19-28.

4. Kazimierz P, Joanna K, Anna H (2010) Biochemistry of Magnesium. J Elementol 15: 601-616

5. Durlach J, Bara M, Guiet-Bara A, Collery P (1986) Relationship between magnesium, cancer and carcinogenic or anticancer metals. Anticancer Res 6 : 1353-1361.

6. Deheinzelin D, Negri EM, Tucci MR, Salem MZ, da Cruz VM, et al. (2000) Hypomagnesemia in critically ill cancer patients: a prospective study of predictive factors. Braz J Med Biol Res 33: 1443-1448.

7. Anghileri LJ (1979) Magnesium concentration variations during carcinogenesis Magnesium Bull 1: 46-48.

8. Anghileri LJ, Collery P, Coudoux P, Durlach J (1981) Experimental relationships between magnesium and cancer. Magnesium Bull 3: 1-5.

9. Anghileri LJ, Heidbreder M, Weiler G, Dermietzel R(1977) Hepatocarcinogenesis by thioacetamide: correlations of histological and biochemical changes, and possible role of cell injury. Exp Cell Biol 45: 34-47.

10. Blondell JW (1980) The anticancer effect of Magnesium. Medical Hypothesis.

11. Whitney RB, Sutherland RM (1972) The influence of calcium, magnesium and cyclic adenosine 3',5'-monophosphate on the mixed lymphocyte reaction. J Immunol 108: 1179-1183.

12. Petitou M, Tuy F, Rosenfeld C, Mishal Z, Paintrand M, et al. (1978) Decreased microviscosity of membrane lipids in leukemic cells: two possible mechanisms. Proc Natl Acad Sci U S A 75: 2306-2310.

13. Hass GM, mccreary PA, Laing GH, Galt RM (1980) Lymphoproliferative and immunologic aspects of magnesium deficiency. Magnesium in Health and disease (2nd International Mg Symposium, Montreal, Canada, 1976) b Eds M. Cantin, M.S. Seelig, Publ. Spectrum Press NY 185-200.

14. Nicholas Calvino DC, Stephen Levine (2001) Vitamin C and Cancer - Storm of Controversy. Cancer 71.

15. Guzmán M (2003) Cannabinoids: potential anticancer agents. Nat Rev Cancer 3: 745-755. 
Citation: Pandey HM, Chaudhary N (2015) To Restrict the Growth of Cancer and Convert it into a Cancer without Disease or Remission by Changing Dietary Patterns. J Cancer Sci Ther 7: 019-030. doi:10.4172/1948-5956.1000320

16. Bifulco M, Laezza C, Gazzerro P, Pentimalli F (2007) Endocannabinoids as emerging suppressors of angiogenesis and tumor invasion (review). Oncol Rep 17: 813-816

17. Bifulco M, Di Marzo V (2002) Targeting the endocannabinoid system in cancer therapy: a call for further research. Nat Med 8: 547-550.

18. Pisanti S (2007) Endocannabinoids as emerging suppressors of angiogenesis and breast tumor growth and metastatic spreading.

19. Mahapatra AK, Mahapatra S (2013) Anticancer role of cannabinoids in glioma.

20. Oaks JL, Gilbert M, Virani MZ, Watson RT, Meteyer CU, et al. (2004) Diclofenac residues as the cause of vulture population decline in Pakistan. Nature 427: 630-633

21. Albert sanchez JL, Reeser HS, Lau PY, Yahiku RE, Willard PJ, et al. (1973) Role of sugars in human neutrophilic phagocytosis; Magie and U.D. Register.

22. Sergei I, Grivennikov, Florian RG, Michael K (2010) Immunity, Inflammation, and Cancer. Cell 140: 833-899.
23. David Servan Schrieber (2007) Anti Cancer- a new way of life. archINFORM: 304

24. Li WW, Li VW, Hutnik M, Chiou AS (2012) Tumor angiogenesis as a target for dietary cancer prevention. J Oncol 2012: 879623.

25. Michael B. Sporn (1999) Prevention of cancer in the next millennium: Report of the Chemoprevention Working Group to the American Association for Cancer Research. Cancer Res 59: 4743-4758.

26. Chansiw N, Pangjit K, Tuntiwechapikul W, Phisalaphong C, Fucharoen S, et al. (2013) Cytotoxicity and Apoptogenic Activity of A Novel Synthetic Iron Chelator 1-(N-Acetyl-6-Aminohexyl)-3-Hydroxy-2-Methylpyridin-4-One (CM1) In Human Leukemic Cells. Vitam Miner 2:114.

27. Toyokuni $S$ (2009) Role of iron in carcinogenesis: cancer as a ferrotoxic disease. Cancer Sci 100: 9-16.

28. Hunt BJ, Bélanger LF (1972) Localized, multiform, sub-periosteal hyperplasia and generalized osteomyelosclerosis in magnesium-deficient rats. Calcif Tissue Res 9: 17-27. 